\title{
State Switching of a Multistable Impacting System using PD- Like Control
}

\author{
Boying Liu ${ }^{1}$, Wai-keung Fung ${ }^{1}$, Yang Liu ${ }^{2}$ \\ ${ }^{1}$ School of Engineering, Robert Gordon University, Aberdeen, United Kingdom, AB10 7GJ \\ Email: \{b.liu4,w.k.fung\}@rgu.ac.uk \\ ${ }^{2}$ College of Engineering, Mathematics and Physical Sciences, University of Exeter, Exeter, UK, EX4 4QF \\ Email: y.liu2@exeter.ac.uk
}

\begin{abstract}
A new PD-like control law is proposed in this paper to control a multistable impacting system. This control law can switch the system from a current, undesired state to a desired one by using the differences of the displacement and velocity between the current and desired states. The control law can control the multistable system without affecting its original dynamics, and its stability is proved by using the Lyapunov direct method. Numerical results are compared with the results obtained by using the intermittent control studied in [1]. The proposed PD-like control shows a better performance in terms of the smoothness of its control signal, which is easier to be implemented in practical applications.
\end{abstract}

\section{Introduction}

Multistability exists in nature and engineering systems in various fields, such as biology [2], electronics [3] and mechanics [4]. In recent years, many researchers have been paying attention on how control of multistability can be achieved with regard to its high sensitivity to system parameters and external perturbation [5]. Control of multistability can enhance system efficiency by switching its state from an undesired state with large amplitude periodic motion to a desired one with small amplitude periodic motion. For example, maintaining small amplitude periodic oscillations in drill-strings can improve the efficiency of drilling, and therefore, extending lifespan of equipment and reducing maintenance cost. Maintaining large amplitude motion for energy harvester can generate more energy and improve its efficiency. In order to control multistable systems, several control methods have been proposed, including steering the system by a feedforward control strategy [6], applying a short pulse to multistable systems [7], and using a pseudo-periodic force to destroy the undesired attractor [8]. These proposed methods are capable of altering an undesired state to a desired state in multistable systems. However, these control laws have limitations. For example, when co-existing attractors in a multistable system are destroyed, its parameters may be affected, and the structure of basins of attraction of the system may become complex. This makes control the system difficult. If the system is under the influence of noise or perturbation, the system may be switched back to undesired state easily. The control law is then switched on and off continuously in high speed and the system will exhibit chattering behaviour. Therefore, it is important to keep the original basin of attraction intact, and thus, guarantee that the system stays in desired state in the presence of noise.

Liu et al. proposed an intermittent control law for state switching in multistable systems [1]. Intermittent control applies an impulse force to the system in order to switch the system to the desired state. However, this control law requires the information of the nonlinearity of the plant model, which is sometimes difficult to acquire accurately. In this paper, a new PD-like control that does not depend on the nonlinearity of the plant is proposed. The proposed control law is simple to be implemented and the basin of attraction of the system will not be changed.

In order to evaluate the performance of this proposed control law, an impact oscillator, which is a typical multistable system, is selected for investigation in this work. It is a simple one degree of freedom system that models the basic mechanism for a variety of engineering systems, such as rotor, gearbox and percussive drilling system [9]. The study of basins of attraction of this system has been extensively reported in literature through numerical analysis and experiment [10-12].

The paper is organised as follows. In Section 2, the mathematical model of the selected multistable system is presented. The proposed PD-like control law and its stability are studied using the Lyapunov direct method in Section 3. In Section 4, the performance of the proposed control law is evaluated using the selected multistable system through simulation. Finally, some concluding remarks are given in Section 5. 


\section{Impact oscillator}

The mathematical model of an impact oscillator is presented as follows. The physical model of the impact oscillator is shown in Figure 1. It includes a mass which is connected to a linear spring and a viscous damper, and the impact oscillator is excited by harmonic force. When the displacement of the mass exceeds the gap between the mass and the secondary spring, impact will occur. Nondimensional model of the impact oscillator is shown below.

$$
\ddot{x}=\Gamma \sin (\omega \tau)-2 \xi \dot{x}-x-\beta(x-e) H(x-e)
$$

Equation 2 shows the process of changing dimensional parameters of the impact oscillator in figure 1 to the nondimensional parameters.

$$
\begin{gathered}
x=\frac{y}{x_{0}}, \quad \tau=\omega_{n} t, \quad \beta=\frac{k_{2}}{k_{1}}, \quad \omega=\frac{\Omega}{\omega_{n}}, \\
\xi=\frac{c}{2 m \omega_{n}}, \quad \Gamma=\frac{a}{x_{0}}, \quad e=\frac{g}{x_{0}}
\end{gathered}
$$

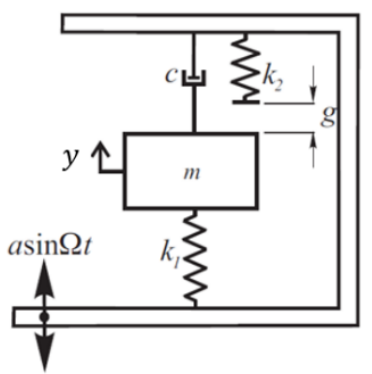

Fig. 1. Schematics of impact oscillator [10]

Two reference values are used for the nondimensional formula. One is the reference distance $x_{0}>0$ and the other is the natural frequency $\omega_{n}=\sqrt{k_{1} / m}$. Moreover, $x$ and $\tau$ are nondimensional displacements of the mass and time separately. $\beta$ is the stiffness ratio, $\omega$ is the frequency ratio and $\xi$ is the damping ratio, whilst $\Gamma$ and $e$ denote the nondimensional amplitude and the gap between mass and the second spring respectively. $H($.$) is the Heaviside step$ function.

\section{The Proposed Control Law}

In this section, we present the proposed PD-like contro law and its stability proof on an impact oscillator.

\subsection{The Governing equations}

In general, when a multistable system is controlled by a state switching control law $u(t)$, its dynamic model is written as follow.

$$
\ddot{x}=f(x, \dot{x})+p(t)+u(t)
$$

After the system is switched to its desired state, its dynamic model becomes.

$$
\ddot{x}_{d}=f\left(x_{d}, \dot{x}_{d}\right)+p(t)
$$

where $x, \dot{x}$ and $x_{d}, \dot{x}_{d}$ are the displacement and velocity of current state and desired state of the system respectively, $f(x, \dot{x})$ and $f\left(x_{d}, \dot{x}_{d}\right)$ model the nonlinearity of the system at current and desired states respectively, $p(t)$ is the external harmonic excitation and $u(t)$ is the control law applied to the system.

Define

$$
\begin{aligned}
& e_{1}=x-x_{d} \\
& e_{2}=\dot{x}-\dot{x}_{d}
\end{aligned}
$$

which are the difference in displacement and velocity between the current and desired trajectories respectively. The proposed PD-like control law is shown as follows:

$$
u(t)=-k_{p} e_{1}-k_{d} e_{2}
$$

where $k_{p}$ and $k_{d}$ are the positive gains of the control law.

When the control law is switched on, the system will be driven to the desired state $\left(x_{d}, \dot{x}_{d}\right)$ within a certain duration of time i.e. $e_{1}$ and $e_{2}$ are zeros and the control input $u(t)$ becomes zero. Thus, the system is switched from the current undesired state to the desired state. This control law can steer dynamical systems without changing the parameters of the system and thus the structure of basin of attraction is not affected. Furthermore, when the system is affected by sensor noise and external disturbance, this control law forces the system to remain in the desired state, or move back to the desired state. Indeed, the control law guides the system by exploiting only the difference between the current state and desired state under the same parameters. Exact knowledge of the nonlinearity of the plants is not required. In order to prevent damage to the actuator within the system, the control input $u(t)$ is bounded by actuator limit $u_{\max }$ and the control law is modified as follows:

$$
u(t)= \begin{cases}-k_{p} e_{1}-k_{d} e_{2} & |u|<u_{\max } \\ \operatorname{sign}(u) \cdot u_{\max } & |u| \geq u_{\max }\end{cases}
$$

\subsection{Stability Analysis}

The stability of the proposed PD-like control law is studied on the selected multistable system using Lyapunov Stability Theorem. The error dynamics after the control law is applied to the system is defined as:

$$
\dot{e}_{2}=f^{*}-k_{p} e_{1}-k_{d} e_{2}
$$

where $f^{*}=f(x, \dot{x})-f\left(x_{d}, \dot{x}_{d}\right)$. Note that the effect of the external harmonic excitation $p(t)$ is cancelled in the error dynamics and it is an autonomous system.

A Lyapunov function is proposed for the error dynamics of the system.

$$
V\left(e_{1}, e_{2}\right)=\frac{1}{2}\left(e_{2}+\lambda e_{1}\right)^{2}+\frac{1}{2}\left(k_{p}+\lambda k_{d}-\lambda^{2}\right) e_{1}{ }^{2}
$$

where $0<\lambda<k_{d}$ so that the Lyapunov function is positive definite. 
Time derivative of the Lyapunov function is given as:

$$
\dot{V}\left(e_{1}, e_{2}\right)=\left(e_{2}+\lambda e_{1}\right) f^{*}+\left(\lambda-k_{d}\right) e_{2}{ }^{2}-\lambda k_{p} e_{1}{ }^{2}(
$$

For the impactor oscillator, $f^{*}$ is defined as,

$$
\begin{aligned}
f^{*}= & -2 \xi e_{2}-e_{1}-\beta[(x-e) H(x-e) \\
& \left.-\left(x_{d}-e\right) H\left(x_{d}-e\right)\right]
\end{aligned}
$$

if

$$
k_{d}>-\xi+\sqrt{\xi^{2}+(1+\beta)}
$$

$\dot{V}\left(e_{1}, e_{2}\right)$ is negative definite and the system converges to the desired trajectory asymptotically. Detailed stability proof is presented in the Appendix.

\section{Numerical Simulation}

In order to evaluate the performance of the proposed control law, MATLAB simulation was conducted using the impact oscillator.

\subsection{Impact oscillator}

Figure 2(a) shows the basin of attraction of the impact oscillator with parameters $(\xi=0.01, \beta=29, e=0.01$, $\Gamma=1.0385$ and $\omega=0.686$ ). There are two co-existing attractors. Figure 2(b) shows the trajectory of the two coexisting attractors, one is a period-2 response with one impact (purple region in figure 2(a) and red line in figure 2(b)) and the other one is a period-2 response with two impacts (blue region in figure 2(a) and black line in figure 2(b)). The process of state switching is shown in blue line in figure 2(b). Figures 2(c) and (d) show respectively the displacement and velocity responses of the system when the proposed control law is applied to the system at around 45 seconds under the control parameters $\left(k_{p}=1\right.$ and $k_{d}=$ 5.5). $k_{d}$ is equal to the minimum value according to equation 12 . The system switches to the desired state within 15 seconds. Moreover, when the system converges to the other state, the control input $u(t)$ becomes 0 , as shown in Figure 2(e).

In order to study the sensibility to the control parameters, more sets of the control parameters are used. Different performances of the control signal are observed. Figure 3(a) shows the maximum peak of nondimensional control force $u(t)$ with different sets of $k_{p}$ and $k_{d}$. In figure 3(a), the maximum peak of control force $u(t)$ varies from 8.89 to 29.47 . The maximum peak of control force $u(t)$ increases with the increasing $k_{d}$. Moreover, the maximum peak of control force $u(t)$ decreases with increasing $k_{p}$. Moreover, the switching duration of different $k_{p}$ and $k_{d}$ are shown in figure 3(b). The switching duration varies from 2.51 seconds to 13.31 seconds. Both of the maximum peak of the control force $u(t)$ and the switching duration are sensitive to the gains in control law. (a)

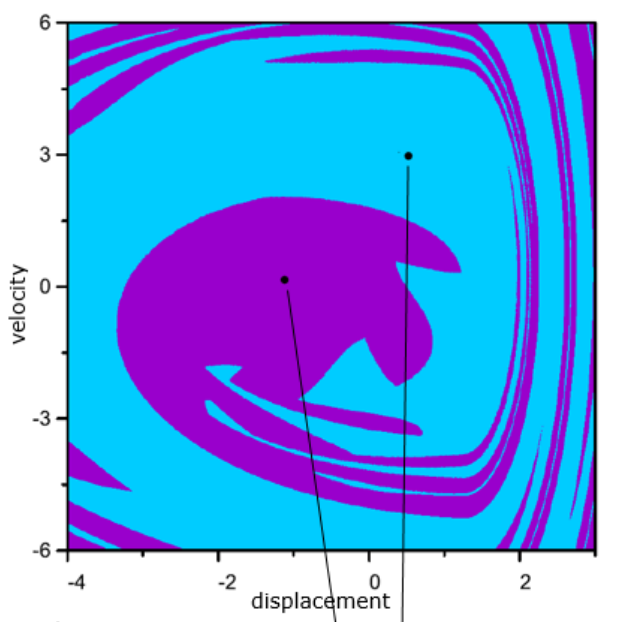

(b)
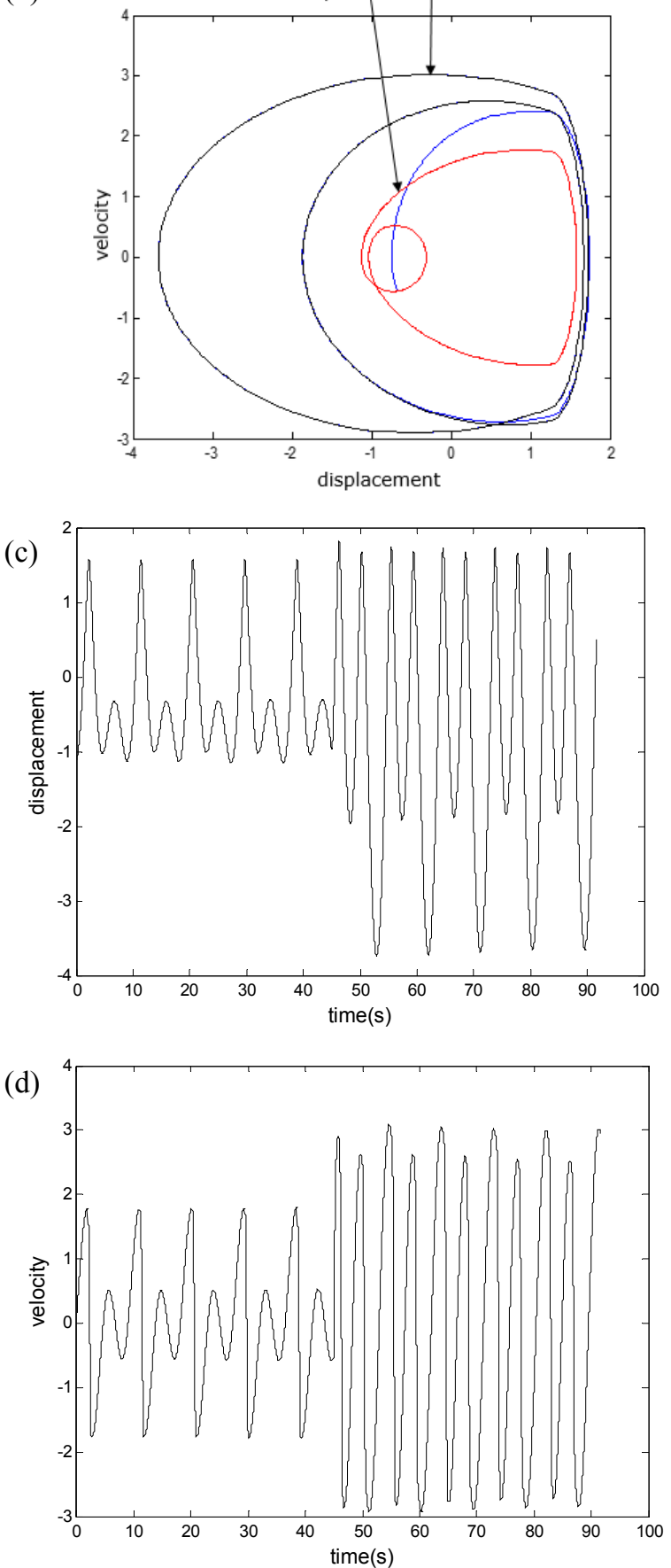


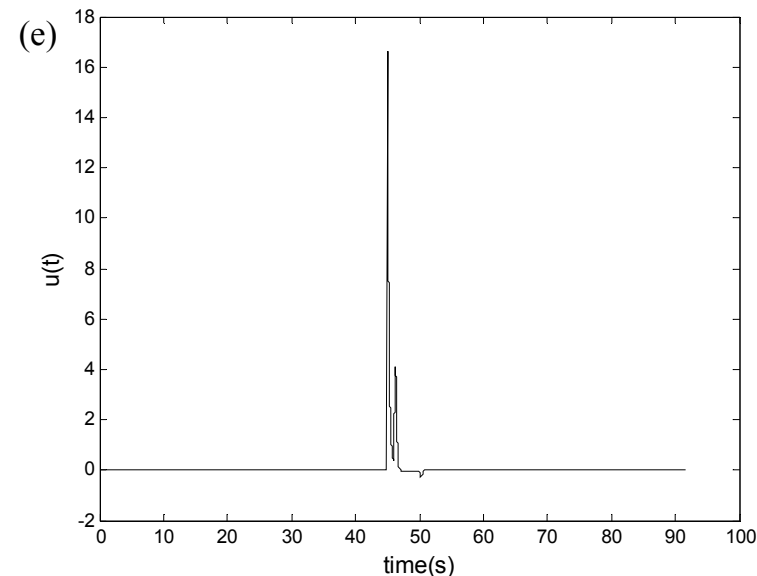

Fig. 2. The simulation result of applying the PD-like control law on an impact oscillator. Parameters of the system: $\xi=0.01$, $\beta=29, e=0.01, \Gamma=1.0385$ and $\omega=0.686$. Parameter of control law: $k_{p}=1$ and $k_{d}=5.5$. (a) Basin of attraction. (b) State trajectories of the two co-existing attractors on phase plane. (c), (d) Displacement and velocity of mass respectively. (e) The applied control force
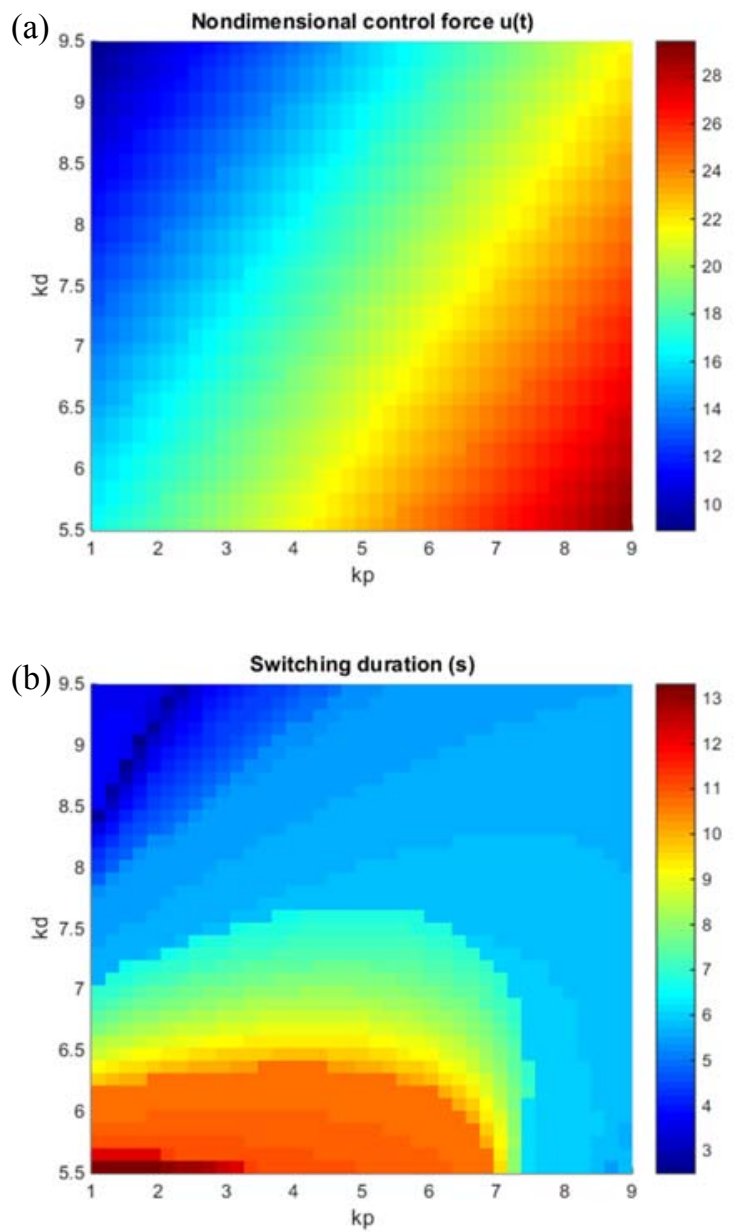

Fig. 3. The simulation result of apply the PD-like control law on impact oscillator with different $k_{p}$ and $k_{d}$. The colour bars show the magnitude of nondimensional control force $u(t)$ and switching duration (a) The nondimensional control force $u(t)$ with different $k_{p}$ and $k_{d}$. (b) The switching duration of the control law with different $k_{p}$ and $k_{d}$

\subsection{Comparison with Intermittent Control}

The intermittent control law proposed in [8] is shown as follows:

$$
\begin{gathered}
\ddot{x}=f(x, \dot{x})+p(t)+u(t) \\
u(t)=\left\{\begin{array}{cc}
0 \quad\left|e_{1}\right|>\delta \\
-f^{*}-k_{p} e_{1}-k_{d} e_{2} & \left|e_{1}\right| \leq \delta
\end{array}\right. \\
f^{*}=f(x, \dot{x})-f\left(x_{d}, \dot{x}_{d}\right) \\
k_{d} \geq \frac{\ln \left[\left|\dot{e}_{1}\left(t_{b}\right)\right| / \epsilon\right]}{\tau}
\end{gathered}
$$

where $\delta$ and $\epsilon$ define the error of the displacement and velocity between the current undesired and desired trajectories respectively and they are adequately small. When $\left|e_{1}\right| \leq \delta$ and $\left|e_{2}\right| \leq \epsilon$, the intermittent control switches on. $t_{b}$ is the first time the system satisfies this condition and $\tau$ is the duration of time when the intermittent control law is on. Moreover, the control parameter $k_{d}$ can be chosen according to equation (14). The proposed PD-like control law looks like the intermittent control law except the nonlinearity term $f^{*}$ is absent.

The performance of the proposed PD-like control law and the intermittent control law are compared by applying them to the impact oscillator, discussed in Section 4.1, with the following parameters:

$$
\delta=1.2, \epsilon=10^{-5}, k_{p}=1 \text { and } k_{d}=5.5
$$

both control laws are applied with the same $k_{p}$ and $k_{d}$ gains. Figure 4 shows the control input $u(t)$ to the impact oscillator when the proposed PD-like control and intermittent control are applied to the impact oscillator under the same system parameters and control parameters. There is no time limit for both control laws or $\tau=+\infty$. In Figure 4, both control laws can switch the system to the desired state. Moreover, the trough magnitude of the control input, generated by the PD-like control law, is smaller than that of the intermittent control law. The control input generated by the PD-like control law has fewer troughs. Furthermore, the PD-like control law takes less time to switch the system from the present state to the desired state than the intermittent control law.

Figure 5 depicts simulation results when the external control input is bounded in both control laws due to actuator limit $u_{\text {max }}$. Equation (15) shows the constrained PD-like control, and equation (16) shows the constrained intermittent control.

$$
\begin{gathered}
u(t)= \begin{cases}-k_{p} e_{1}-k_{d} e_{2} & |u|<u_{\max } \\
\operatorname{sign}(u) \cdot u_{\max } & |u| \geq u_{\max }\end{cases} \\
\mathrm{u}(t)= \begin{cases}0 & \left|e_{1}\right|>\delta \\
-f^{*}-k_{p} e_{1}-k_{d} e_{2} & \left|e_{1}\right| \leq \delta,|u|<u_{\max } \\
\operatorname{sign}(u) \cdot u_{\max } & \left|e_{1}\right| \leq \delta,|u| \geq u_{\max }\end{cases}
\end{gathered}
$$

where $u_{\max }$ is the bound of the absolute magnitude of control input $u(t)$.

Both control laws have the same control parameters and they are applied at the same time to the impact oscillator. Both of them are capable of switching the system into the desired state with various $u_{\max }$. For example, when $u_{\max }=5$, both control laws exhibit very 
similar behaviours in $u(t)$ shortly after application of the control action. Overall, the control input generated by the PD-like control law has fewer peaks and troughs than those generated by intermittent control, and it takes less time to complete state switching using the PD-like control laws.

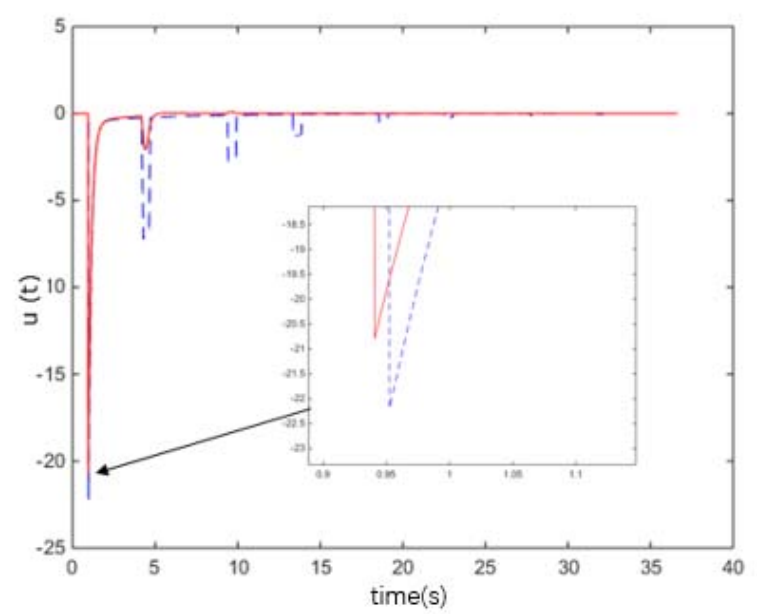

Fig. 4. Control input $u(t)$ to the impact oscillator using intermittent control law (blue dashed line) and PD-like control law (red solid line). System parameters: $\xi=0.01, \beta=29, e=0.01$, $\Gamma=1.0385$ and $\omega=0.686$. Control parameters: $\delta=1.2, \epsilon=10^{-5}$ $k_{p}=1$ and $k_{d}=5.5$. The inset shows the minimum of the control input generated by two control laws.

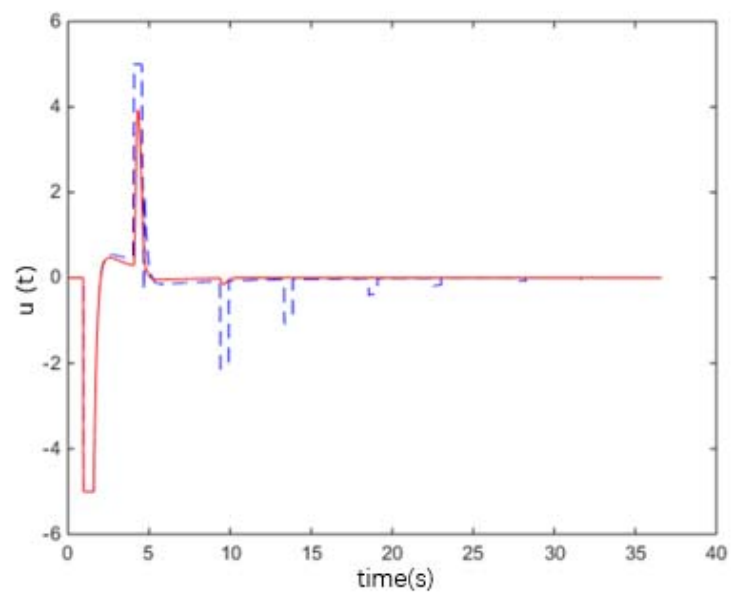

Fig. 5. Simulation results of the impact oscillator with intermittent control law (blue dashed line) and PD-like control law (red solid line). System parameters: $\xi=0.01, \beta=29, e=0.01$, $\Gamma=1.0385$ and $\omega=0.686$. Control parameters: $u_{\max }=5, \delta=1.2$, $\epsilon=10^{-5}, k_{p}=1$ and $k_{d}=5.5$.

Figure 6 shows the simulation results of the control input $u(t)$ when the constrained value of the external control input is very small $\left(u_{\max }=0.5\right)$. The intermittent control law fails to switch the impact oscillator to the desired state. Figure 6(a) shows that the control input $u(t)$ oscillates with high frequency and does not stop. On the other hand, the suggested PD-like control law can switch the system to the desired state in 35 seconds. Obviously, the process of state switching takes a longer time when small actuator limits compared to Figures $4\left(u_{\max }=\infty\right)$ and $5\left(u_{\max }=5\right)$.
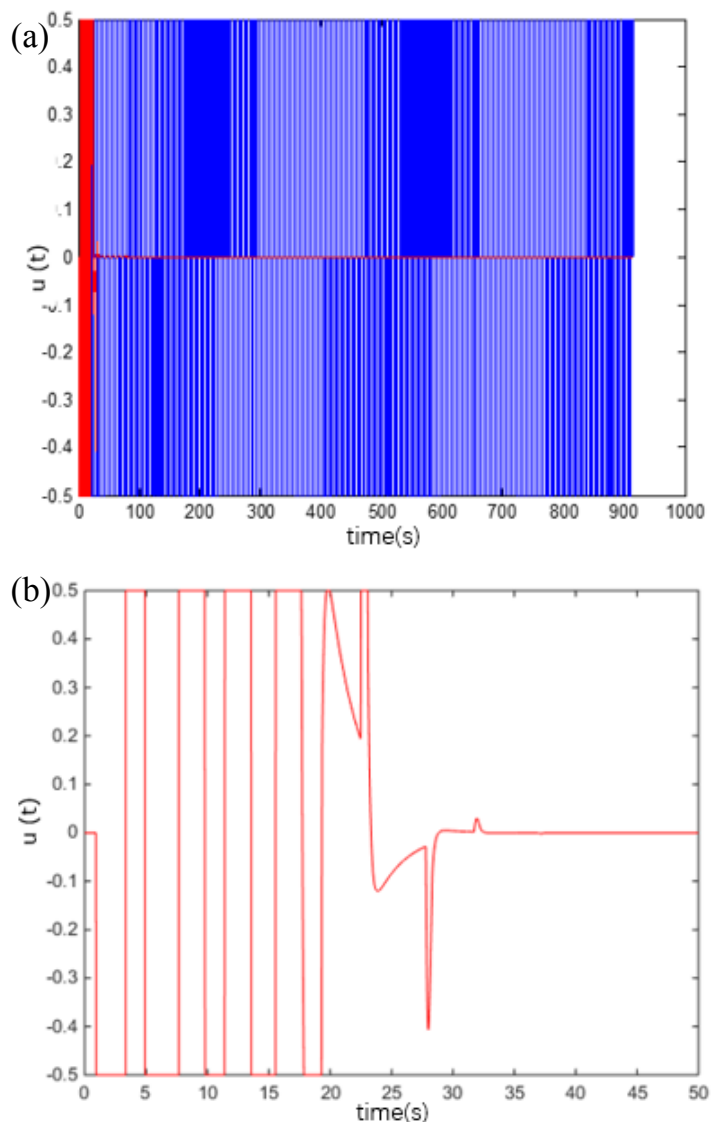

(c)

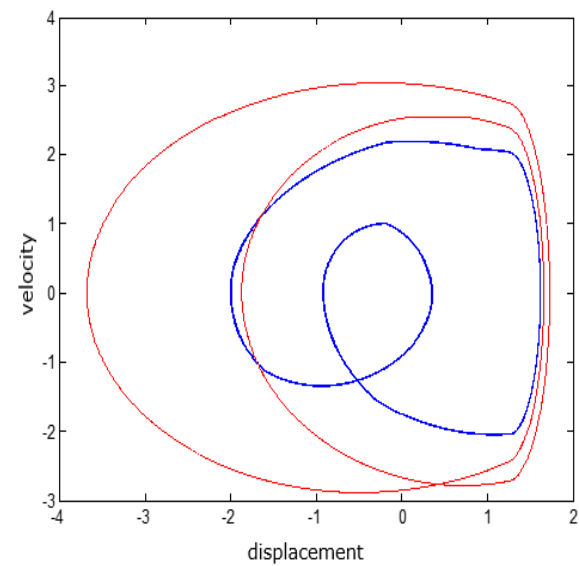

Fig. 5. Simulation results of the impact oscillator with the intermittent control law (blue line) and the PD-like control law (red line). System parameters: $\xi=0.01, \beta=29, e=0.01, \Gamma=1.0385$ and $\omega=0.686$. Control parameters: $u_{\max }=0.5, \delta=1.2, \epsilon=10^{-5}$, $k_{p}=1$ and $k_{d}=5.5$. (a) Control input generated by the two control laws. (b) Detail of control input generated by the PD-like control. (c) State trajectories of response on the phase plane after applying the two control laws.

\section{Conclusion and Future Work}

A PD-like control law was proposed to switch multistable systems to their desired states, without knowing exact knowledge of their nonlinearity, and without destroying the structure of the basin of attraction of the system. Numerical simulations using an impact oscillator demonstrate that the proposed control law can switch impact oscillator from its current, undesired state to the desired state successfully. Moreover, the control input 
generated by the proposed PD-like control is smoother than that generated by the intermittent control. Constrained control force is considered to avoid exceeding actuator limit. The smaller the control force limit, the longer the switching duration.

The peak value of control force $u(t)$ and switching duration of control law are sensitive to the control gains. Smoothness of the control force $u(t)$ can be optimised by minimising the number of peaks, the peak value in $u(t)$ and the switching duration through finding the optimal $k_{p}$ and $k_{d}$ gains and switching time. Some of these objectives are conflicting and multi-objective optimisation of the smoothness of $u(t)$ will be investigated.

\section{References}

[1] Y. Liu, M. Wiercigroah, J. Ing, E. Pavlovskaia, Intermittent control of coexisting attractors, Philos. Trans. R. Soc. (2012)

[2] J. Foss, A, Longtin, B. Mensour, J. Milton, Multistability and Delayed Recurrent Loops, Phys. Rev. Lett, 76, 708-711 (1996)

[3] J. Maurer, A.Libchaber, Effect of the Prandtl number on the onset of turbulence in liquid-He-4, J. de Phys. Lett., 41, 515-518 (1980)

[4] J.M.T Thompson, H.B. Stewart, Nonlinear Dynamics and Chaos, (1986)

[5] A.N. Pisarchik, U. Feudel, Control of Multistability, Phys. R, 540, 167-218 (2014)

[6] D.W.M Veldman, R.H.B. Fey, H. Zwart, Impulsive steering between coexisting stable periodic solutions with an application to vibrating plates, J. Comput. Nonlin. Dyn. (2016)

[7] K. Kaneko, Clustering, coding, switching, hierarchical ordering, and control in a network of chaotic elements, Phys D, 41, 137-172 (1990)

[8] J. Guckenheimer, P. Holms, Nonlinear Oscillations, Dynamical Systems and Bifurcation of Vector Fields, Springer-Verlag, (1983)

[9] Y. Liu, J.P. Chavez, Controlling coexisting attractor of an impacting system via linear augmentation, Phys. D, 348, 1-11 (2017)

[10] J. Ing, E. Pavlovskaia, M. Wiercigroah, S. Banerjee, Experimental study of impact oscillator with one-side elastic constraint, Philos. Trans. R. Soc. (2007)

[11] J. Ing, E. Pavlovskaia, M. Wiercigroah, S. Banerjee, Bifurcation analysis of an impact oscillator with a onesided elastic constraint near grazing, Phys. D, 239 (2010) [12] J. Ing, Near Grazing Dynamics of Piecewise Linear Oscillators, Ph.D. dissertation, School of Engineering, University of Aberdeen, (2008)

\section{Appendix}

The stability proof of the proposed PD-like control law on impact oscillator is presented.

Given a Lyapunov function

$$
V\left(e_{1}, e_{2}\right)=\frac{1}{2}\left(e_{2}+\lambda e_{1}\right)^{2}+\frac{1}{2}\left(k_{p}+\lambda k_{d}-\lambda^{2}\right) e_{1}^{2}
$$

Where $0<\lambda<k_{d}$ so that the Lyapunov function is a positive definite.

In order to prove the $\dot{V}\left(e_{1}, e_{2}\right)$ is negative definite. Four case are considered

$$
\begin{aligned}
& \text { 1. } x<e \text { and } x_{d}<e \\
& \begin{aligned}
\dot{V}\left(e_{1}, e_{2}\right) & =\left(\lambda-k_{d}\right) e_{2}{ }^{2}-\lambda k_{p} e_{1}{ }^{2} \\
& -\left(e_{2}+\lambda e_{1}\right)\left(2 \xi e_{2}+e_{1}\right)
\end{aligned}
\end{aligned}
$$

a. When $e_{1}$ and $e_{2}$ have the same sign, $\dot{V}\left(e_{1}, e_{2}\right)$ is negative definite.

b. When $e_{1}$ and $e_{2}$ have different signs and $\left|e_{1}\right|>$ $\left|e_{2}\right|$. There exists a $\lambda$ which makes $\left(e_{2}+\lambda e_{1}\right)\left(2 \xi e_{2}+\right.$ $\left.e_{1}\right)>0$ and thus $\dot{V}\left(e_{1}, e_{2}\right)$ is negative definite.

c. When $e_{1}$ and $e_{2}$ are different signs and $\left|e_{1}\right|<\left|e_{2}\right|$, There is a bound for $k_{d}: k_{d}>1-2 \xi$ to guarantee $\dot{V}\left(e_{1}, e_{2}\right)$ is negative definite.

$$
\begin{aligned}
& \text { 2. } x>e \text { and } x_{d}>e \\
& \qquad \begin{aligned}
\dot{V}\left(e_{1}, e_{2}\right) & =\left(\lambda-k_{d}\right) e_{2}{ }^{2}-\lambda k_{p} e_{1}^{2} \\
& -\left(e_{2}+\lambda e_{1}\right)\left[2 \xi e_{2}+(1+\beta) e_{1}\right]
\end{aligned}
\end{aligned}
$$

a. When $e_{1}$ and $e_{2}$ have the same sign, $\dot{V}\left(e_{1}, e_{2}\right)$ is negative definite.

b. When $e_{1}$ and $e_{2}$ have different signs and $\left|e_{1}\right|>$ $\left|e_{2}\right|$. There exists a $\lambda$ which make sure that $\left(e_{2}+\right.$ $\left.\lambda e_{1}\right)\left[2 \xi e_{2}+(1+\beta) e_{1}\right]>0$ and thus $\dot{V}\left(e_{1}, e_{2}\right)$ is negative definite.

c. When $e_{1}$ and $e_{2}$ are different signs and $\left|e_{1}\right|<\left|e_{2}\right|$, There is a bound for $k_{d}: k_{d} \geq-\xi+\sqrt{\xi^{2}+(1+\beta)}$ to ensure that $\dot{V}\left(e_{1}, e_{2}\right)$ is negative definite.

3. $x>e$ and $x_{d}<e$

$$
\begin{aligned}
\dot{V}\left(e_{1}, e_{2}\right) & =\left(\lambda-k_{d}\right) e_{2}{ }^{2}-\lambda k_{p} e_{1}{ }^{2} \\
& -\left(e_{2}+\lambda e_{1}\right)\left[2 \xi e_{2}+e_{1}+\beta(x-e)\right]
\end{aligned}
$$

Since $x>e$ and $x_{d}<e, 0<x-e<e_{1}$

a. When $e_{2}>0, \dot{V}\left(e_{1}, e_{2}\right)$ is negative definite.

b. When $e_{2}<0$ and $\left|e_{1}\right|>\left|e_{2}\right|$, There exists a $\lambda$ to guarantee that $\left(e_{2}+\lambda e_{1}\right)\left[2 \xi e_{2}+e_{1}+\beta(x-e)\right]>0$ and thus $\dot{V}\left(e_{1}, e_{2}\right)$ is negative definite.

c. When $e_{2}<0$ and $\left|e_{1}\right|<\left|e_{2}\right|$. There is a bound for $k_{d}: \quad k_{d} \geq-\xi+\sqrt{\xi^{2}+(1+\beta)}$ to make sure that $\dot{V}\left(e_{1}, e_{2}\right)$ is negative definite.

$$
\begin{aligned}
& \text { 4. } x<e \text { and } x_{d}>e \\
& \begin{aligned}
\dot{V}\left(e_{1}, e_{2}\right) & =\left(\lambda-k_{d}\right) e_{2}{ }^{2}-\lambda k_{p} e_{1}{ }^{2} \\
& -\left(e_{2}+\lambda e_{1}\right)\left[2 \xi e_{2}+e_{1}-\beta\left(x_{d}-e\right)\right]
\end{aligned}
\end{aligned}
$$

Since $x<e$ and $x_{d}>e, e_{1}<e-x_{d}<0$

a. When $e_{2}<0, \dot{V}\left(e_{1}, e_{2}\right)$ is negative definite.

b. When $e_{2}>0$ and $\left|e_{1}\right|>\left|e_{2}\right|$, There exists a $\lambda$ to ensure that $\left(e_{2}+\lambda e_{1}\right)\left[2 \xi e_{2}+e_{1}-\beta\left(x_{d}-e\right)\right]>0$ and thus $\dot{V}\left(e_{1}, e_{2}\right)$ are negative definite.

c. When $e_{2}>0$ and $\left|e_{1}\right|<\left|e_{2}\right|$. There is a bound for $k_{d}: \quad k_{d} \geq-\xi+\sqrt{\xi^{2}+(1+\beta)}$ to make sure that $\dot{V}\left(e_{1}, e_{2}\right)$ is negative definite. 